\title{
Análise do modelo de Holt-Winters aplicado a uma série histórica de dados com tendência e sazonalidade
}

\author{
Feroni, R. C. ${ }^{*} ;$ Andreão, W. L. ${ }^{2}$ \\ ${ }^{1}$ Departamento de Engenharias e Tecnologia, Universidade Federal do Espírito Santo, São Mateus, ES, Brasil. \\ ${ }^{2}$ Programa de Pós-Graduação em Saneamento, Meio Ambiente e Recursos Hídricos, Universidade Federal de Minas \\ Gerais, Belo Horizonte, MG, Brasil. \\ *e-mail: rita.feroni@ufes.br
}

\begin{abstract}
Resumo
Modelos de suavização exponencial são amplamente utilizados para previsões a partir de séries temporais de dados. Quando a série apresenta nível, tendência e sazonalidade, o modelo de Holt-Winters pode ser satisfatoriamente aplicado. Neste trabalho, para a avaliação do modelo, foi utilizada uma série genérica, porém que se assemelha aos dados de demanda real de produtos que apresentam demanda sazonal. A partir da análise de erros, os resultados mostram que o modelo de Holt-Winters aditivo obteve melhor performance comparado ao modelo multiplicativo. As previsões dos dois modelos conseguiram acompanhar satisfatoriamente o comportamento dos dados reais durante o período analisado.
\end{abstract}

\begin{abstract}
Exponential smoothing models are widely used for predictions from time series of data. When the series presents level, trend and seasonality, the Holt-Winters model can be satisfactorily applied. In this work, to evaluate the model, a generic series was used, however, which resembles data of real demand for products that present seasonal demand. From the error analysis, the results show that the Holt-Winters additive model obtained better performance compared to the multiplicative model. The predictions of the two models were able to satisfactorily fit the real data during the analyzed period.
\end{abstract}

Keywords (Palavras chaves): série temporal, previsão, suavização exponencial, modelo de Holt-Winters.

\section{Introdução}

A análise de séries temporais é usada em muitas aplicações no ramo da engenharia, como análise econômica, previsão de demanda, previsões meteorológicas, dentre outras. Este tipo de análise exige o conhecimento de valores passados da variável que se quer prever, sendo essa coleção de valores tomados em instantes específicos de tempo, com igual espaçamento. A expectativa é de que o padrão observado nos valores passados, forneça informação adequada para a previsão de valores futuros da variável [1]. Se o período coberto for suficientemente longo, o padrão da variável resultante permite distinguir comportamentos associados a série temporal como, efeito de tendência, efeito sazonal e variações irregulares ou ao acaso.
Fatores como o comportamento da variável no tempo, habilidade do programador, tipo de aplicação, disponibilidade de dados, tempo, recurso computacional e horizonte de planejamento, dentre outros, irão nortear o programador a aplicar o melhor modelo baseado em séries temporais. Neste contexto, os modelos de suavização exponencial são amplamente utilizados para previsão devido a sua simplicidade, facilidade de ajuste e boa precisão [2].

Em casos reais, o comportamento relacionado a série nem sempre pode ser visto por análise de tabelas ou análise gráfica, dessa forma, comparações entre os modelos e seus dados de entradas são necessárias a partir de uma análise de erro para determinar o melhor modelo. Dessa forma, o objetivo deste trabalho é 
investigar a utilização e as entradas do modelo HoltWinters [3], a partir de uma série histórica de dados. A série refere-se aos dados de vendas de agasalhos de uma empresa do setor de vestuário, porém devido as restrições de divulgação das informações coletadas, e pelas características da série serem representativas de vários setores que possuem suas vendas influenciadas pelas estações do ano, a série de dados será tratada como uma série genérica que apresenta tendência e sazonalidade.

\section{Métodos de Suavização exponencial}

Três variações básicas de modelos de suavização exponencial podem ser citadas: suavização exponencial de primeira ordem [4], aplicada satisfatoriamente quando a série temporal se mantém constante sobre um nível médio [1,2]; modelo de Holt [5], que pode ser utilizado, de maneira satisfatória, em séries temporais com tendência linear [1,2]; e o modelo de Holt-Winters [3], aplicado satisfatoriamente a séries temporais que exibem tendência e sazonalidade [2]. Este último método será visto com mais detalhes.

\subsection{Modelo Holt- Winters}

O modelo de Holt-Winters é uma expansão do método Holt [5] desenvolvida por Winters [3] e se divide em dois grupos: modelo de Holt-Winters multiplicativo, onde a amplitude da variação sazonal aumenta ou diminui como função do tempo, dado pelas Equações (1) a (4) [6], no Quadro 1, e modelo de Holt-Winters aditivo, onde a amplitude da variação sazonal é constante ao longo do tempo, dado pelas equações de (5) a (8). Nas equações aparece um componente relacionado à previsão $\left(\widehat{z}_{t+k}\right)$, ao nível $(L)$, a tendência $(T)$ e a sazonalidade $(S)$.

2.2 Análise das variáveis de entrada dos modelos e metodologia

Os valores das constantes de suavização $\alpha, \beta$ e $\gamma$ são arbitrários. A determinação dos melhores valores pode ser feita utilizando uma análise como o desvio absoluto médio (MAD) Equação (9), o erro percentual absoluto médio (MAPE), Equação (10) ou o erro quadrático médio (MSE), Equação (11), também apresentadas no Quadro 1. Assim, seleciona-se um valor inicial aleatoriamente, a partir do qual as previsões são geradas. Os parâmetros que minimizarem, por exemplo o MSE, são utilizados no modelo final.

Quadro 1: Equacionamento do modelo e análise de erro.

\begin{tabular}{|c|c|}
\hline Holt-Winters multiplicativo & Eq. \\
\hline$\hat{z}_{t+k}=\left(L_{t}+k T_{t}\right) S_{t-s+k}$ & $(1)$ \\
\hline$L_{t}=\alpha \frac{Z_{t}}{S_{t-s}}+(1-\alpha)\left(L_{t-1}+T_{t-1}\right)$ & (2) \\
\hline$T_{t}=\beta\left(L_{t}-L_{t-1}\right)+(1-\beta) T_{t-1}$ & (3) \\
\hline$S_{t}=\gamma \frac{Z_{t}}{L_{t}}+(1-\gamma) S_{t-s}$ & (4) \\
\hline Holt-Winters aditivo & Eq. \\
\hline$\hat{z}_{t+k}=\left(L_{t}+k T_{t}\right)+S_{t-s+k}$ & (5) \\
\hline$L_{t}=\alpha\left(z_{t}-S_{t-s}\right)+(1-\alpha)\left(L_{t-1}+T_{t-1}\right)$ & (6) \\
\hline$T_{t}=\beta\left(L_{t}-L_{t-1}\right)+(1-\beta) T_{t-1}$ & (7) \\
\hline$S_{t}=\gamma\left(z_{t}-L_{t)}+(1-\gamma) S_{t-s}\right.$ & (8) \\
\hline$M A D=\frac{\sum_{t=1}^{n}\left|z_{t-\hat{z}_{t}}\right|}{n}$ & (9) \\
\hline$M A P E=\left(\frac{\sum_{t=1}^{n}\left|\left(z_{t-} \hat{z}_{t}\right) / z_{t}\right|}{n}\right) \times 100$ & $(10)$ \\
\hline$M S E=\frac{\sum_{t=1}^{n}\left(z_{t-} \hat{z}_{t}\right)^{2}}{n-1}$ & $(11)$ \\
\hline$L_{s}=\frac{1}{s}\left(z_{1}+z_{2}+\cdots+z_{s}\right)$ & $(12)$ \\
\hline$T_{s}=\frac{1}{s}\left(\frac{Z_{s+1-} Z_{1}}{s}+\frac{Z_{s+2-} Z_{2}}{s}+\cdots+\frac{Z_{s+s-} Z_{s}}{s}\right)$ & $(13)$ \\
\hline$S_{1}=\frac{Z_{1}}{L_{s}}, S_{2}=\frac{Z_{2}}{L_{s}}, \ldots, S_{s}=\frac{Z_{s}}{L_{s}}$ & $(14)$ \\
\hline$S_{1}=z_{1}-L_{s}, S_{2}=z_{2}-L_{s}, \ldots, S_{s}=z_{s}-L_{s}$ & $(15)$ \\
\hline \multicolumn{2}{|c|}{$\begin{array}{l}\text { Subscritos: } \\
t: \text { refere-se ao período; } \\
s: \text { comprimento da sazonalidade; } \\
k: \text { períodos a frente; } \\
\text { Variáveis, } \\
\hat{z}_{t+k}: \text { previsão para } k \text { períodos a frente; } \\
\hat{z}: \text { previsão de demanda; } \\
z: \text { valor real observado na série; } \\
L: \text { estimativa para o nível da série; } \\
T: \text { estimativa da tendência da série; } \\
S: \text { estimativa de sazonalidade da série; } \\
\alpha, \beta \text { e } \gamma: \text { constantes de suavização (valores entre } 0 \\
\text { e } 1) . \\
n: \text { número de observações associados a previsão. }\end{array}$} \\
\hline
\end{tabular}

Para o modelo de Holt-Winters são necessários valores iniciais de componentes para nível, tendência e sazonalidade, para que os cálculos sejam iniciados. Para o componente sazonal, é necessário ao menos uma estação completa de observações, $s$ períodos. A partir disso, as estimativas do nível e da tendência são feitas, no período $s$ definido para o componente 
sazonal. Em Makridakis et al. [6], pode-se encontrar as estimativas iniciais de cada componente, para o modelo multiplicativo: nível (Equação 12), tendência (Equação 13) e sazonalidade (Equações 14) e para o modelo aditivo: nível (Equação 12), tendência (Equação 13) e sazonalidade (Equações 15), sendo que a tendência requer duas estações completas para sua estimativa.

A fim de investigar a aplicabilidade do modelo de HoltWinters, a série histórica de dados mostrada na Figura 1 é analisada.

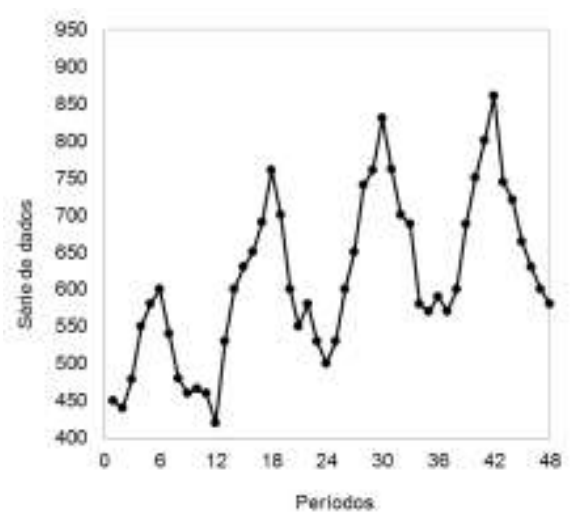

Figura 1: Série histórica de dados.

\section{Resultados e Discussões}

A série de dados escolhida para análise deve ser primeiramente analisada. Neste caso, a série foi cuidadosamente escolhida para que pudesse apresentar nível, tendência e sazonalidade para análise do modelo. A partir de tal análise, foi possível verificar o nível no qual as observações flutuam, a tendência ascendente e a presença do fator sazonal, que ocorre a cada doze períodos (s). Pela análise já é possível inferir que possivelmente o melhor método de suavização exponencial será de Holt-Winters.

As equações para cada modelo assim como os valores inicias foram calculados conforme formulação mostrada no Quadro 1. A solução ótima para cada modelo foi escolhida a partir da ferramenta "solver" do ambiente MS-Excel ${ }^{\circledR}$ para determinar os parâmetros $\alpha, \beta$ e $\gamma$ (constantes de suavização) que minimizam o MSE.
Dados de entrada e uma comparação relacionada aos erros associados aos modelos podem ser vistos na Tabela 1. O valor da constante $\beta$ igual a zero pode significar que os valores iniciais para tendência foram bem estimados e nenhuma atualização foi necessária para se obter o MSE menor possível.

Tabela 1: Entradas dos modelos e análise de erro.

\begin{tabular}{|c|c|c|c|c|c|c|}
\hline & $\alpha$ & $\beta$ & $\gamma$ & MAD & MAPE & MSE \\
\hline$M^{*}$ & 0,66 & 0 & 0,25 & 34,10 & 1,26 & 2070,321 \\
\hline$A^{* *}$ & 0,97 & 0 & 0,58 & 33,77 & 0,96 & 1956,56 \\
\hline
\end{tabular}

A fim de comparar os valores previstos com os dados da série histórica e realizar uma previsão para os próximos 12 períodos, a Figura 2 foi gerada. A partir dela, verifica-se um satisfatório acompanhando dos modelos com relação aos dados observados. A Figura 2 em análise conjunta com a Tabela 1, nos permitem afirmar que o modelo mais confiável de previsão para o conjunto de dados é o Holt-Winters aditivo (M.H.W.A), por possuir o menor MAD, MAPE e MSE. O valor do MAPE de 0,96 para o modelo aditivo mostra que em média, a previsão de erro é menor que $1 \%$.

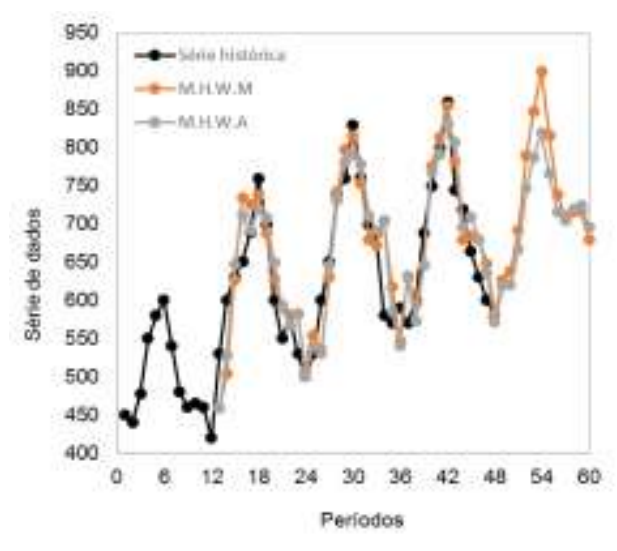

Figura 2: Comparação de previsões.

\section{Conclusões}

Realizar previsões não se resume apenas em aplicar modelos em séries de dados e gerar resultados, mas deve-se compreender o comportamento da série de dados e buscar através de observações críticas a escolha do melhor modelo. 


\section{Encontro Científico de Física Aplicada}

\section{Blucher}

No presente trabalho, foi realizada uma análise da aplicação do modelo de suavização exponencial proposto por Holt-Winters aplicado a uma série histórica de dados que apresenta tendência e sazonalidade. A série escolhida é genérica, porém seu comportamento se assemelha a dados históricos de demanda, por exemplo, de produtos como biquínis, sorvetes, agasalhos, que possuem, em geral, comportamento sazonal quando a análise é feita anual com dados reais mensais.

Os resultados obtidos foram bastante satisfatórios sendo o modelo de Holt-Winters aditivo o mais adequado a ser aplicado a série de dados apresentada, com a previsão acompanhando satisfatoriamente os dados reais e apresentando menores erros associados, quando comparado com o modelo multiplicativo. Observa-se que mesmo mostrando bons resultados, a previsão é uma ferramenta que contém erros. Por esta razão, as metodologias de previsão quantitativas devem sempre estar associada a técnicas qualitativas de análise, o que está bastante relacionado à experiência do programador.

\section{Referências}

[1] MOREIRA, D. A. Administração da produção e operações. 2. ed. São Paulo: Cengage Learning, 2016.

[2] PELLEGRINI, F.R.; FOLIATTO, F.S. Revista Produção, v. 11, n. 1, p. 43-64, 2001.

[3] WINTERS, P. R. Management Science, v. 6, n. 3, p.324-342, 1960.

[4] BROWN, R.G. Statistical Forecasting for Inventory Control. New York: McGraw-Hill, 1959.

[5] HOLT, C.E. International Journal of Forecasting, v. 20, p.5-10, 2004.

[6] MAKRIDAKIS, S. et al. Forecasting Methods and Applications. 3. ed. New York: John Wiley \& Sons, 1998. 